J. M. M. Evans · S. A. Ogston •

A. Emslie-Smith · A. D. Morris

\title{
Risk of mortality and adverse cardiovascular outcomes in type 2 diabetes: a comparison of patients treated with sulfonylureas and metformin
}

Received: 27 September 2005 / Accepted: 25 November 2005 / Published online: 9 March 2006

(C) Springer-Verlag 2006

\begin{abstract}
Aims/hypothesis: The aim of this study was to evaluate the risk of adverse cardiovascular outcomes in patients with type 2 diabetes newly treated with sulfonylureas and metformin. Subjects and methods: The Diabetes Audit and Research in Tayside Scotland (DARTS) diabetes information system and the Medicines Monitoring Unit (MEMO) dispensed prescribing database for the population of Tayside, Scotland (400,000 people) were employed. Patients newly prescribed with oral hypoglycaemic agents between 1994 and 2001 were classified into five study cohorts according to the treatment received: metformin only, sulfonylureas only, sulfonylureas added to metformin, metformin added to sulfonylureas, and both drugs simultaneously. In Cox regression analyses, we estimated relative risks for all-cause mortality, cardiovascular mortality and cardiovascular hospital admission for patients in the five study cohorts, with metformin monotherapy as the reference group. Results: Of the 5,730 study patients, 1,000 died during a maximum of 8 years follow-up. Patients in the sulfonylureas only cohort had increased risks of mortality and cardiovascular mortality, with unadjusted relative risks of 3.12 (95\% CI 2.54-3.84) and 3.71 (95\% CI 2.64-5.22), respectively. After adjusting for differences between groups (age, sex, duration of diabetes, blood pressure, cholesterol, $\mathrm{HbA}_{1 \mathrm{c}}$, smoking, previous hospital admission, treatment
\end{abstract}

J. M. M. Evans $(\bowtie) \cdot$ S. A. Ogston

Section of Public Health, Division of Community Health

Sciences, University of Dundee Medical School,

Dundee, UK

e-mail: j.m.m.stansfield@dundee.ac.uk

Tel.: +44-1382-420108

Fax: +44-1382-420101

A. Emslie-Smith

The Mill Practice,

Dundee, UK

\section{A. D. Morris}

Division of Medicine and Therapeutics, University of Dundee Medical School,

Dundee, UK with cardiovascular medication), these relative risks were 1.43 (95\% CI 1.15-1.77) and 1.70 (95\% CI 1.18-2.45), respectively. Patients in the combination cohorts had significantly increased risks of cardiovascular hospital admission, as well as increased risks of mortality and cardiovascular mortality. Conclusions/interpretation: In this cohort study of patients newly treated with oral hypoglycaemic agents, those treated with sulfonylureas only, or combinations of sulfonylureas and metformin, were at higher risk of adverse cardiovascular outcomes than those treated with metformin alone.

Keywords Cardiovascular risk - Metformin . Sulfonylureas

Abbreviations DARTS: Diabetes Audit and Research in Tayside Scotland - MEMO: Medicines Monitoring Unit . UGDP: University Group Diabetes Program - UKPDS: United Kingdom Prospective Diabetes Study · NHS: National Health Service - OHA: oral hypoglycaemic agent . ICD-9/10: International Classification of Diseases, 9th and 10th revisions - AIIRA: angiotensin II receptor antagonist

\section{Introduction}

A Canadian study recently re-ignited the debate over the cardiovascular effects of oral hypoglycaemic agents (OHAs) [1]. Using routine datasets that included 1,150 users of metformin therapy and 3,033 users of sulfonylureas, this observational study identified a decreased risk of mortality and cardiovascular mortality with metformin therapy [1]. Could this indicate a cardiotoxic effect of sulfonylurea therapy? Sulfonylureas are at the core of pharmacotherapy in type 2 diabetes. Up to a third of all patients are prescribed sulfonylureas at some point (although metformin is usually the first drug of choice in overweight and obese patients).

The safety of sulfonylureas in type 2 diabetes has been an ongoing issue since the results of the University Group Diabetes Program (UGDP) were published over 30 years ago. The increased risk of cardiovascular mortality 
associated with the sulfonylurea tolbutamide led to its withdrawal in the United States, although the results of the UGDP study have been much criticised [2]. It has been suggested that sulfonylureas have a direct deleterious effect on the heart but this is outweighed by the beneficial effects mediated by their metabolic action [3].

In the United Kingdom Prospective Diabetes Study (UKPDS), a trial designed to compare different therapies in type 2 diabetes, no adverse effect of sulfonylureas on cardiovascular outcomes was noted [4]. However, the lack of a beneficial effect of sulfonylureas on macrovascular outcomes, despite improved glycaemic control and a reduced risk of microvascular outcomes, could be inferred as further evidence of direct cardiovascular toxicity. The complicated analysis strategy in the UKPDS makes interpretations difficult [5]. Another worrying result was a $96 \%$ increase in diabetes-related deaths in sulfonylureatreated patients with addition of metformin [6]. Observational studies have shown increased risks associated with a combination of metformin and sulfonylureas, but have been unable to account fully for underlying differences between patients in different therapy groups [7, 8]. In contrast, a more recent study found no increased risk of mortality among patients who were prescribed sulfonylureas and metformin in combination, compared with those prescribed either drug as monotherapy [9].

We have carried out a retrospective cohort study to test the hypothesis that, compared with new users of metformin, new users of sulfonylureas are at no increased risk of cardiovascular outcomes. Because patients in different therapy groups are likely to have different underlying risks of morbidity and mortality, we used detailed clinical information on patients to adjust for potential confounding factors between cohorts.

\section{Subjects and methods}

We used the data resources of the Diabetes Audit and Research in Tayside, Scotland (DARTS) / the Medicines Monitoring Unit (MEMO) Collaboration for this study [10, 11]. This collaboration has developed the record linkage of multiple, routinely collected data sets in the population of Tayside (approximately 400,000 people). The UK National Health Service (NHS) provides healthcare to virtually all residents of Tayside. Record linkage is enabled by the use of a unique ten-digit identifier (known as the Community Health Index Number or CHI) allocated to residents when they register with an NHS general practitioner, which is then anonymised for record linkage research.

The MEMO dataset [10] contains computerised patientspecific data on every prescription dispensed in Tayside from January 1993. A record of every prescription for metformin and sulfonylureas dispensed to patients with diabetes is contained within MEMO, with details of drug name, dose, date of prescription and prescribed regimen. Data on all other prescriptions dispensed to diabetic patients (insulin, cardiovascular medication) are also available.
The DARTS dataset contains detailed clinical information on every patient diagnosed with diabetes in Tayside from January 1993 [11]. Data collection for this dataset includes collation and validation of routine data, and ongoing retrieval of information directly from medical records in primary care by a dedicated team of research nurses. Clinical information is collected according to the national clinical dataset for the care of diabetic people in Scotland, and includes diabetes type, date of diagnosis, duration, therapy, $\mathrm{HbA}_{1 \mathrm{c}}$, presence (and date) of microvascular and macrovascular diabetic complications and cardiovascular risk factors (BMI, smoking and blood pressure).

Using the DARTS database, we identified all Tayside residents who were diagnosed with type 2 diabetes prior to December 2001. This did not include patients who were diagnosed under the age of 35 years and had a requirement for insulin within 90 days, and were therefore defined as patients with type 1 diabetes. We then identified all those who were newly treated with OHAs during the study period (January 1994 to December 2001). Patients with records of OHA prescriptions in 1993 were ineligible as they were not new OHA users during the study period. We also excluded patients who were prescribed insulin either before or within 90 days of their first OHA prescription.

Five cohorts were defined:

1. Metformin monotherapy: patients treated with metformin only throughout the study period, or those treated with metformin with sulfonylureas added later (at which point they were censored). Their index date was the date of their first metformin prescription.

2. Sulfonylureas monotherapy: patients treated with sulfonylureas only throughout the study period, or those treated with sulfonylureas with metformin added later (at which point they were censored). Their index date was the date of their first sulfonylureas prescription.

3. Combination 1: patients treated with metformin with sulfonylureas added later. Their index date was the date of their first sulfonylureas prescription.

4. Combination 2: patients treated with sulfonylureas with metformin added later. Their index date was the date of their first metformin prescription.

5. Both: treatment with both sulfonylureas and metformin on the same day. Their index date was the date of their first prescription for both.

The patients were followed prospectively from their index date until censoring, death or the end of the study period. The primary outcome measures were all-cause mortality, cardiovascular mortality and hospital admission where the primary diagnosis code was for a cardiovascular diagnosis. Mortality was ascertained from death certification records from the Registrar General with cause of death coded according to International Classification of Diseases, 9th and 10th revisions (ICD-9/10) [12,13]. If there was any ICD-9/10 code for cardiovascular disease in the underlying cause of death field on the death certificate, this was defined as a cardiovascular death. Hospital admissions were ascertained from Scottish Morbidity Record 1 data. These are computerised records of all hospital admissions 
of Tayside residents with ICD-9/10 diagnostic codes, in which there are up to six diagnosis codes. If the primary diagnosis code was a code for cardiovascular disease, this was defined as a cardiovascular admission.

We compared survival between cohorts using KaplanMeier survival plots. Cox regression analyses were carried out to estimate the relative risks of each outcome for patients in the study cohorts (with the metformin monotherapy cohort as the reference group). Survival times were censored if patients left Tayside, or at the end of the study period. Where cardiovascular admission was the outcome variable, deaths were treated as censored observations.

The following confounding variables were investigated: sex, duration of diabetes at index date, age at index date, Carstairs deprivation category (an area-based measure of material deprivation [14]), and whether patients had a previous cardiovascular admission between 1980 and their index date. The first record of smoking status that was available after the index date was noted. Average BMI, blood pressure, $\mathrm{HbA}_{1 \mathrm{c}}$ and cholesterol were determined from records that were available for the duration of the study period. For each cohort, we also determined the proportion of patients who had received prescriptions for any of four drug types: aspirin, statins, beta-adrenoceptor blocking drugs (beta-blockers) and ACE inhibitors or angiotensin-II receptor antagonists (AIIRAs). Continuous covariates were categorised into quartile groups where appropriate. Patients with missing values were assigned to separate categories. Covariates were included in the final models if they were individually statistically significant $(p<0.05)$, or known risk factors for cardiovascular events.

\section{Results}

In the Tayside region, 6,089 patients with type 2 diabetes were newly prescribed with OHAs during the study period. Of these, 196 were excluded as they were prescribed insulin either before or just after their first OHA prescription. Of the remaining 5,893 patients, 5,730 with full demographic and clinical information were available for analysis.

The metformin monotherapy cohort comprised 2,286 patients, of which 985 were later prescribed sulfonylureas. Of the 3,331 patients in the sulfonylureas monotherapy cohort, 1,252 were later prescribed metformin. Therefore, there were 985 and 1,252 patients in the two combination cohorts, denoted as Combination 1 and 2, respectively. There were 113 patients who were treated with both drugs initially. The demographic characteristics and cardiovascular risk factors of patients in the five study cohorts are presented in Table 1. Patients in the sulfonylureas monotherapy cohort had the highest mean age, and this cohort had highest proportion of males.

There were 1,000 deaths during the study period, of which 411 were defined as primary cardiovascular mortality. In total, 1,033 patients were admitted to hospital during the study period with an ICD-9/10 code for a cardiovascular diagnosis as the primary diagnosis code. The number of patients in each study cohort who had these outcomes are presented in Table 2, with unadjusted risk ratios. We found that $4.7 \%$ of patients in the metformin monotherapy cohort died (35.5\% cardiovascular deaths), compared with $17.9 \%$ of patients in the sulfonylureas monotherapy cohort (42.4\% cardiovascular deaths). Figure 1 shows cumulative mortality (1-survival) derived from Kaplan-Meier survival curves for patients in the five cohorts. Patients in the

Table 1 Demographic characteristics and cardiovascular risk factors of the patients in the five study cohorts

\begin{tabular}{|c|c|c|c|c|c|}
\hline$z$ & $\begin{array}{l}\text { Metformin } \\
\text { monotherapy }\end{array}$ & $\begin{array}{l}\text { Sulfonylureas } \\
\text { monotherapy }\end{array}$ & Combination 1 & Combination 2 & Both \\
\hline Total & 2,286 & 3,331 & 985 & 1,252 & 113 \\
\hline Women $(\%)^{\mathrm{b}}$ & 48.8 & 43.7 & 53.0 & 44.9 & 50.4 \\
\hline Mean age (years) ${ }^{b}$ & 60.2 & 65.9 & 61.2 & 63.6 & 64.0 \\
\hline Carstairs $^{\mathrm{a}, \mathrm{b}}(\%$ ranks $1,2 / 3,4 / 5-7)$ & $22.6 / 40.9 / 36.5$ & $24.9 / 45.8 / 29.3$ & $23.2 / 39.7 / 37.1$ & $24.3 / 46.1 / 29.5$ & $25.7 / 40.4 / 33.9$ \\
\hline Mean diabetes duration ${ }^{\mathrm{b}}$ (years) & 2.7 & 4.6 & 4.4 & 4.8 & 8.8 \\
\hline Previous CV hospitalisation ${ }^{\mathrm{b}}(\%)$ & 29.2 & 34.0 & 36.9 & 34.1 & 18.6 \\
\hline Smoking $^{\mathrm{b}}$ (\% never/current/ex) & $47.0 / 28.0 / 20.6$ & $43.4 / 27.4 / 18.6$ & $48.7 / 27.4 / 40.7$ & $47.4 / 28.9 / 20.5$ & $48.7 / 22.1 / 18.6$ \\
\hline Mean $\mathrm{HbA}_{1 \mathrm{c}}{ }^{\mathrm{b}}$ & 7.7 & 7.8 & 8.1 & 8.2 & 8.0 \\
\hline Mean $\mathrm{BMI}^{\mathrm{b}}$ & 32.9 & 28.6 & 33.2 & 30.2 & 30.0 \\
\hline Mean systolic blood pressure ${ }^{\mathrm{b}}$ & 142.9 & 142.3 & 143.2 & 142.8 & 142.8 \\
\hline Mean diastolic blood pressure ${ }^{\mathrm{b}}$ & 80.9 & 79.0 & 81.3 & 80.3 & 79.4 \\
\hline Mean cholesterol & 5.20 & 5.16 & 5.24 & 5.23 & 5.10 \\
\hline Using aspirin ${ }^{\mathrm{b}}(\%)$ & 43.1 & 50.2 & 47.0 & 49.7 & 48.7 \\
\hline Using statins ${ }^{\mathrm{b}}(\%)$ & 47.1 & 38.5 & 50.6 & 46.4 & 44.2 \\
\hline Using beta-blockers ${ }^{\mathrm{b}}(\%)$ & 32.9 & 27.5 & 31.0 & 26.7 & 21.2 \\
\hline Using ACE inhibitors/AIIRAs ${ }^{\mathrm{b}}(\%)$ & 53.0 & 47.3 & 56.5 & 54.2 & 54.0 \\
\hline
\end{tabular}

${ }^{\mathrm{a}}$ Highest ranks denote least material deprivation; ${ }^{\mathrm{b}}$ statistically significant $(p<0.01)$ in $\chi^{2}$ tests and ANOVA 
Table 2 The 5-year survival rates and unadjusted risk ratios (with 95\% CIs) for mortality, cardiovascular mortality and hospital admission for the patients in the study cohorts

\begin{tabular}{|c|c|c|c|c|c|c|c|}
\hline \multirow[t]{2}{*}{ Cohort } & \multicolumn{3}{|l|}{ Mortality } & \multicolumn{2}{|c|}{ Cardiovascular mortality } & \multicolumn{2}{|c|}{ Cardiovascular admission } \\
\hline & 5 -year survival rate & $n$ & $\mathrm{RR}(95 \% \mathrm{CI})$ & $n$ & $\mathrm{RR}(95 \% \mathrm{CI})$ & $n$ & $\mathrm{RR}(95 \% \mathrm{CI})$ \\
\hline Metformin monotherapy & $0.81(0.79-0.83)$ & 107 & 1.00 & 38 & 1.00 & 229 & 1.00 \\
\hline Sulfonylureas monotherapy & $0.68(0.67-0.69)$ & 597 & $3.12(2.54-3.84)$ & 253 & $3.71(2.64-5.22)$ & 567 & $1.11(0.61-2.03)$ \\
\hline Combination 1 & $0.84(0.82-0.87)$ & 113 & $1.86(1.43-2.42)$ & 42 & $1.94(1.25-3.01)$ & 92 & $1.42(0.80-2.54)$ \\
\hline Combination 2 & $0.82(0.74-0.90)$ & 167 & $2.07(1.62-2.64)$ & 72 & $2.50(1.69-3.71)$ & 133 & $2.15(1.21-3.81)$ \\
\hline Both & $0.84(0.76-0.92)$ & 16 & $1.74(1.02-2.94)$ & 6 & $1.82(0.77-4.31)$ & 12 & $1.22(0.68-2.21)$ \\
\hline
\end{tabular}

$R R$, Risk ratio

sulfonylureas monotherapy cohort had at least three times the risk of mortality and cardiovascular mortality compared with patients in the metformin monotherapy cohort, but no significant increased risk of cardiovascular hospital admission. Patients in the combination cohorts were at increased risk of mortality, cardiovascular mortality and cardiovascular hospital admission.

Table 3 presents risk ratios for these outcomes, adjusted for all confounding factors. The only covariate not to be included in the final model was Carstairs deprivation category. Patients in the sulfonylureas monotherapy cohort had an increased risk of mortality and cardiovascular mortality compared with the metformin monotherapy cohort, even after adjusting for demographic and clinical differences between the groups, but no significant increased risk of cardiovascular hospital admission. Adjusted risks for mortality and cardiovascular mortality were lower than unadjusted risks, suggesting that the baseline differences between the groups were partially due to differences in the distribution of underlying confounding factors. Patients in the combination cohorts had even higher risks of mortality, cardiovascular mortality and cardiovascular hospital admission, compared with the metformin monotherapy cohort, although the adjusted risks were higher than the unadjusted risks.
The risks of mortality and cardiovascular mortality both increased with increasing age, with females at a slightly reduced risk. Patients who had a previous cardiovascular admission were also at increased risk, and ex- or never smokers were at lower risk than current smokers. Patients in the highest quartile of diastolic blood pressure had a slightly higher risk (although this was not statistically significant), but this was not evident for systolic blood pressure. There was evidence of trends for increased risk with increasing diabetes duration, but decreasing BMI and $\mathrm{HbA}_{1 \mathrm{c}}$. Patients for whom data were unavailable were also at high risk. The risk of cardiovascular hospital admission differed somewhat from the other outcomes. There seemed to be decreasing risk with increasing blood pressure and cholesterol, and increased risk with increasing BMI and $\mathrm{HbA}_{1 \mathrm{c}}$. Adjusted risk ratios for cardiovascular hospital admission were higher than unadjusted risk ratios.

Use of any of the drug types was associated with increased hospital admission. There was a reduced risk of mortality and cardiovascular mortality in statin users, but an increased risk of cardiovascular mortality associated with aspirin use. Patients who used ACE inhibitors or AIIRAs had a reduced risk of mortality.

During the follow-up period, 565 patients commenced insulin treatment. However, the adjusted risk ratios for this
Fig. 1 Cumulative mortality rates (with standard errors at yearly intervals) in five study cohorts: 1. Metformin monotherapy: patients treated with metformin only (blue line). 2. Sulfonylureas monotherapy: patients treated with sulfonylureas only (yellow line). 3. Combination 1: patients treated with metformin with sulfonylureas added later (pink line). 4. Combination 2: patients treated with sulfonylureas with metformin added later (brown line). 5. Both: treatment with both sulfonylureas and metformin on the same day (purple line)

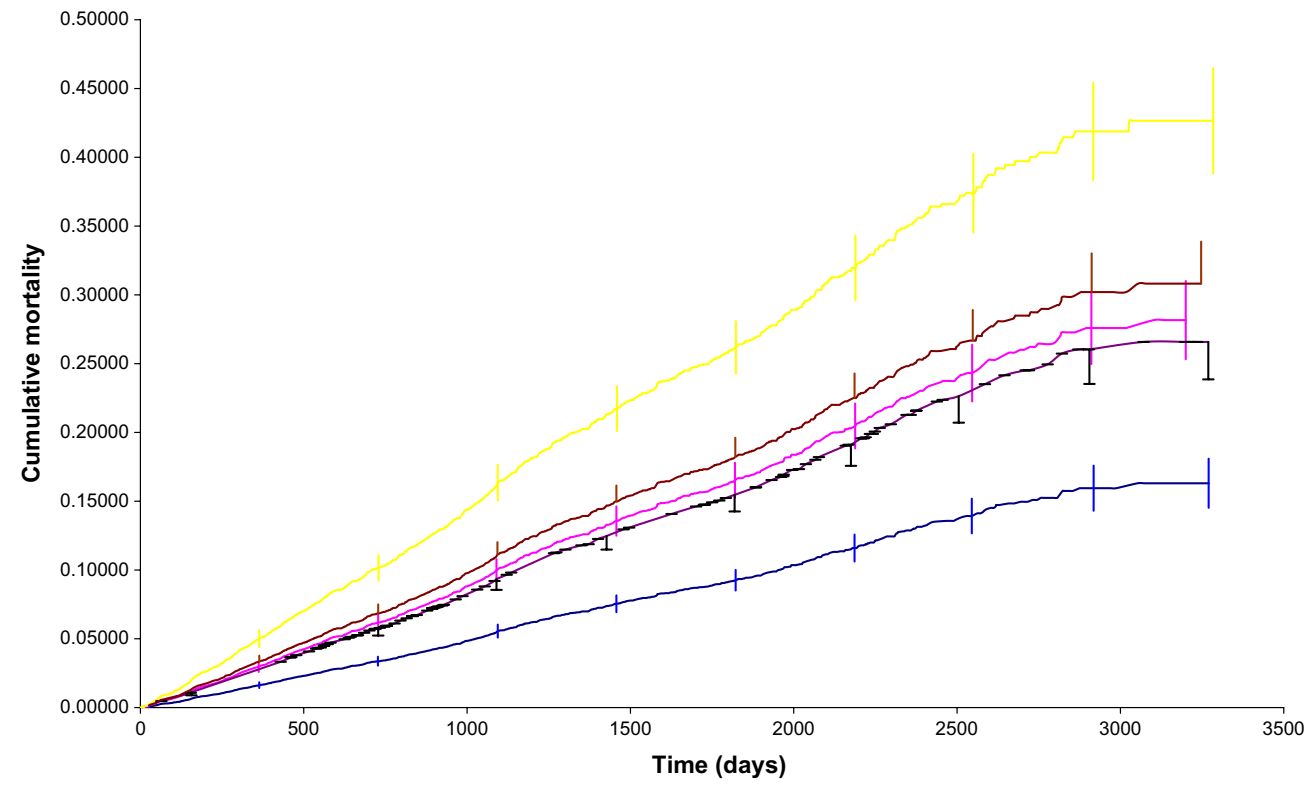


Table 3 Adjusted risk ratios (with 95\% CIs) for all covariates

\begin{tabular}{|c|c|c|c|}
\hline & \multicolumn{3}{|c|}{ Adjusted risk ratios $(95 \% \mathrm{CI})$} \\
\hline & Mortality & Cardiovascular mortality & Cardiovascular admission \\
\hline \multicolumn{4}{|l|}{ Cohort } \\
\hline Metformin monotherapy & 1.00 & 1.00 & 1.00 \\
\hline Sulfonylureas monotherapy & $1.43(1.15-1.77)$ & $1.70(1.18-2.45)$ & $1.30(0.71,2.40)$ \\
\hline Combination 1 & $2.47(1.88-3.25)$ & $2.29(1.45-3.61)$ & $1.86(1.03,3.35)$ \\
\hline \multicolumn{4}{|l|}{ Age } \\
\hline $0-44$ years & 1.00 & 1.00 & 1.00 \\
\hline 45-64 years & $3.98(2.22-7.16)$ & $3.98(1.45-10.93)$ & $2.25(1.47,3.44)$ \\
\hline $65-84$ years & $8.31(4.63-14.92)$ & $7.67(2.80-21.04)$ & $2.42(1.57-3.75)$ \\
\hline $65+$ years & $11.56(6.26-21.25)$ & $10.41(3.64-29.75)$ & $3.65(2.16-6.16)$ \\
\hline Quartile 3 & $1.09(0.91-1.30)$ & $1.06(0.79-1.43)$ & $0.95(0.79-1.13)$ \\
\hline Quartile 4 & $2.00(0.63-6.36)$ & $1.18(0.90-1.56)$ & $0.89(0.74-1.07)$ \\
\hline \multicolumn{4}{|l|}{ Sex } \\
\hline Male & 1.00 & 1.00 & 1.00 \\
\hline Female & $0.83(0.73-0.95)$ & $0.90(0.73-1.11)$ & $0.90(0.79-1.03)$ \\
\hline \multicolumn{4}{|l|}{ Previous hospital admission } \\
\hline No & 1.00 & 1.00 & 1.00 \\
\hline Yes & $1.98(1.73-2.27)$ & $2.06(1.66-2.56)$ & $1.97(1.72-2.25)$ \\
\hline \multicolumn{4}{|l|}{ Diastolic BP } \\
\hline Quartile 1 (lowest) & 1.00 & 1.00 & 1.00 \\
\hline Quartile 2 & $0.99(0.81-1.20)$ & $0.90(0.66-1.22)$ & $0.84(0.70-1.00)$ \\
\hline \multicolumn{4}{|l|}{ Smoking } \\
\hline Current & 1.00 & 1.00 & 1.00 \\
\hline Ex & $0.72(0.58-0.89)$ & $0.75(0.54-1.05)$ & $0.82(0.68-0.99)$ \\
\hline Never & $0.74(0.61-0.90)$ & $0.75(0.55-1.03)$ & $0.90(0.76-1.07)$ \\
\hline Not known & $1.25(0.90-1.74)$ & $1.67(1.01-2.76)$ & $1.19(0.80-1.78)$ \\
\hline \multicolumn{4}{|l|}{ Cholesterol } \\
\hline Quartile 1 (lowest) & 1.00 & 1.00 & 1.00 \\
\hline Quartile 2 & $1.39(0.96-2.00)$ & $1.24(0.69-2.23)$ & $0.97(0.77-1.22)$ \\
\hline Quartile 3 & $1.37(0.94-1.98)$ & $1.19(0.66-2.15)$ & $0.75(0.59-0.97)$ \\
\hline Quartile 4 & $2.00(1.41-2.84)$ & $2.09(1.21-3.58)$ & $0.92(0.72-1.18)$ \\
\hline Not known & $1.94(1.44-2.62)$ & $1.87(1.18-2.98)$ & $0.74(0.60-0.90)$ \\
\hline \multicolumn{4}{|l|}{ BMI } \\
\hline Quartile 1 (lowest) & 1.00 & 1.00 & 1.00 \\
\hline Quartile 2 & $0.84(0.69-1.03)$ & $0.93(0.68-1.27)$ & $1.04(0.86-1.26)$ \\
\hline Quartile 3 & $0.74(0.59-0.93)$ & $0.84(0.59-1.20)$ & $1.09(0.90-1.33)$ \\
\hline Quartile 4 & $0.73(0.56-0.95)$ & $0.82(0.55-1.24)$ & $1.13(0.91-1.40)$ \\
\hline Not known & $1.10(0.83-1.46)$ & $1.32(0.85-2.05)$ & $1.94(1.39-2.71)$ \\
\hline \multicolumn{4}{|l|}{$\mathrm{HbA}_{1 \mathrm{c}}$} \\
\hline Quartile 1 (lowest) & 1.00 & 1.00 & 1.00 \\
\hline Quartile 2 & $0.63(0.51-0.79)$ & $0.63(0.44-0.89)$ & $0.92(0.76-1.12)$ \\
\hline Quartile 3 & $0.61(0.49-0.76)$ & $0.75(0.54-1.04)$ & $1.11(0.92-1.34)$ \\
\hline
\end{tabular}


Table 3 (continued)

\begin{tabular}{llll}
\hline & \multicolumn{1}{l}{ Adjusted risk ratios $(95 \%$ CI) } & Cardiovascular admission \\
\cline { 2 - 4 } & Mortality & Cardiovascular mortality & $1.18(0.98-1.42)$ \\
\hline Quartile 4 & $0.74(0.61-0.91)$ & $0.85(0.62-1.16)$ & $2.01(1.50-2.68)$ \\
Not known & $2.30(1.86-2.85)$ & $2.15(1.53-3.03)$ & 1.00 \\
Aspirin use & & & $2.82(2.40-3.30)$ \\
No & 1.00 & 1.00 & 1.00 \\
Yes & $1.02(0.89-1.18)$ & $1.54(1.23-1.92)$ & $1.38(1.20-1.59)$ \\
Statins & & & 1.00 \\
No & 1.00 & 1.00 & $1.16(1.02-1.32)$ \\
Yes & $0.53(0.45-0.63)$ & $0.60(0.46-0.77)$ & 1.00 \\
Beta-blockers & 1.00 & 1.00 & $1.88(1.63-2.16)$ \\
No & $0.95(0.81-1.10)$ & $0.96(0.76-1.20)$ & \\
Yes & 1.00 & 1.00 & $1.04(0.83-1.29)$ \\
ACE inhibitors/AIIRA & $0.85(0.74-0.97)$ & & \\
No & &
\end{tabular}

group were not different from those for the overall study population (results not shown). 1,947 patients received routine diabetes care in hospital clinics. Results for this group were not different from those for patients treated in primary care (results not shown).

\section{Discussion}

In this study, patients with type 2 diabetes who were newly treated with sulfonylureas were at higher risk of mortality and cardiovascular mortality than those who were newly treated with metformin, although there was no increased risk of cardiovascular hospital admission. The adjusted risk ratios were $1.43(95 \%$ CI $1.15-1.17)$ and 1.70 (95\% CI 1.18-2.45) for mortality and cardiovascular mortality, respectively. Our study is consistent with earlier studies that found patients treated with metformin were at lower cardiovascular risk but were unable to adjust for cardiovascular risk at baseline [1]. For example, the risk ratio for overall mortality for patients treated with metformin compared with sulfonylureas was 0.60 (95\% CI 0.48 0.74) in the Canadian study [1].

The key issue is whether this increased risk is the result of drug therapy, or whether patients prescribed sulfonylureas have a higher baseline cardiovascular risk than those in the monotherapy cohort. Severity of diabetes will clearly influence initial drug choice. This was indeed suggested by increased mean age and duration of diabetes in the sulfonylureas monotherapy cohort, and a higher proportion of males, patients with a previous cardiovascular hospital admission and aspirin users. As expected, patients in the sulfonylureas cohort had a lower BMI than those in the metformin cohort. However, the study suggests that sulfonylureas cannot always be used as recommended given that the mean BMI in this cohort was above $25 \mathrm{~kg} / \mathrm{m}^{2}$.

Adjustment for all potential confounding variables (including BMI) resulted in reduced risk estimates, but patients in the sulfonylureas monotherapy cohort were still at higher risk of mortality and cardiovascular mortality than those in the metformin cohort. These increased risks were observed for all patients, regardless of location of care.

Patients in the combination cohorts had even higher risks of mortality and cardiovascular mortality compared with those in the metformin cohort, even after adjusting for underlying differences between groups. Combination cohort patients also had an increased risk of cardiovascular hospital admission. These results conflict with those from a previous study that, using the UK General Practice Research Database [9], found no increased risk of mortality in combination cohorts compared with patients prescribed drugs singly. However, it is important to recognise that these cohorts represent a heterogeneous mix of patients, with widely varying levels of exposure to sulfonylureas and metformin. It is also possible that prescribing practice may have changed over time, with patients now more likely to be prescribed metformin or combination therapy at an earlier stage of disease than previously, therefore overestimating the risk associated with sulfonylureas in the later years of the study. However, we did not find evidence of such changing practice in our data, with mean duration of diabetes remaining similar for each cohort throughout the years of the study.

The most important question is whether we have adequately adjusted for differences in underlying cardiovascular risk between cohorts. Adjustment for a wide range of potential confounding variables was made, including age, sex, BMI, blood pressure, cholesterol, duration of diabetes, smoking, $\mathrm{HbA}_{1 \mathrm{c}}$ and, very importantly, previous cardiovascular admission. As expected, current smoking, previous cardiovascular admission, increasing age and increasing cholesterol levels were all significant predictors of mortality and cardiovascular mortality, thereby providing evidence for the validity of our study. Patients for whom smoking status and cholesterol were unknown were also at increased risk. This is plausible if such patients 
were not regularly attending clinics and clinical data were therefore incomplete. The inconsistent results for blood pressure are more difficult to explain, although there is some evidence of increased risk among patients with the highest diastolic blood pressure. Patients taking statins were at reduced risk.

The risk factors for cardiovascular hospital admission were different from those for the other outcomes. For example, use of any of the drug types was associated with increased hospital admission; perhaps reflecting a lower threshold for admission in patients already taking cardiovascular medication. Patients with a higher BMI or $\mathrm{HbA}_{1 \mathrm{c}}$ were also more likely to be admitted. An important caveat is that cardiovascular hospital admission is probably the least reliable of the three outcomes, with many selective factors likely to determine whether patients are admitted to hospital and whether they are assigned a primary cardiovascular diagnosis code. In contrast, mortality was ascertained from a validated national database.

It has been argued [15] that the results of the Canadian study [1] might support a cardioprotective effect of metformin rather than an increased cardiovascular risk among users of sulfonylureas, possibly derived from unrecognised differences between therapy groups (there was very little clinical information available for patients [15]). It is therefore important to note that the risks associated with sulfonylureas in our study are in line with what would be expected in a diabetic population, despite being higher than those for the metformin cohort. All-cause mortality of $28 \%$ and cardiovascular mortality of $12 \%$ over a seven-year follow-up are comparable with $24 \%$ and $11 \%$ over 5 years in the Canadian study, and 3\% per year among middle-aged Finnish men [16]. This is consistent with the theory that it may be metformin that is cardioprotective [15] (rather than sulfonylureas being cardiotoxic). The absolute risk of mortality is surprisingly low in the metformin cohort ( $8 \%$ over 7 years), and the proportion of cardiovascular deaths is less; thus, our study does provide some supporting evidence for this theory.

We carried out this study using well-validated data sources. Identification of study cohorts relied upon records of prescriptions dispensed, and identification of study outcomes relied upon national datasets. We are confident in the accuracy of the data, whilst acknowledging the difficulties of ascertaining (and adjusting for) cardiovascular risk using routine data sources. We also acknowledge the limitation of observational research, in that there can be no control for underlying differences between study cohorts. However, while it is possible that increased risks in the sulfonylureas monotherapy cohort and combination cohort are due to residual confounding or other unknown differences at baseline, the consistently higher risks that we have identified, with results that are similar to previous studies [1], suggest that this issue does warrant further investigation. But, as Sasali and Leahy argue [15], we are not yet in a position to advocate cautious prescribing of sulfonylureas to patients at high cardiovascular risk until further corroborating evidence is available.

Acknowledgements This study was funded by Diabetes UK (ref RD03/0002595). We thank members of the DARTS Steering Group for their support. We would also like to thank the support staff of the DARTS/MEMO Collaboration who facilitated this work, notably, P. Thompson for the provision of data.

\section{References}

1. Johnson JA, Simpson SH, Majumdar SR, Toth EL (2002) Decreased mortality associated with the use of metformin compared with sulfonylurea monotherapy in type 2 diabetes. Diabetes Care 25:2244-2248

2. Seltzer HS (1972) A summary of criticisms of the findings of the University Group Diabetes Program. Diabetes 21:976-979

3. Leibowitz G, Cerasi E (1996) Sulphonylurea treatment of NIDDM patients with cardiovascular disease: a mixed blessing? Diabetologia 39:503-514

4. United Kingdom Prospective Diabetes Study (UKPDS) Group (1998) Intensive blood glucose control with sulphonylureas or insulin compared with conventional treatment and risk of complications in patients with type 2 diabetes (UKPDS 33). Lancet 352:837-853

5. Nathan DM (1998) Some answers, more controversy, from UKPDS. Lancet 352:832-833

6. United Kingdom Prospective Diabetes Study (UKPDS) Group (1998) Effect of intensive blood-glucose control with metformin on complications in overweight patients with type 2 diabetes (UKPDS 34). Lancet 352:854-865

7. Olsson J, Lindberg G, Gottsater M et al (2000) Increased mortality in type II diabetic patients using sulphonylurea and metformin in combination: a population-based observational study. Diabetologia 43:558-560

8. Fisman EZ, Tenenbaum A, Boyko V et al (2001) Oral antidiabetic treatment in patients with coronary disease: timerelated increased mortality on combined glyburide/metformin therapy over a 7.7 year follow-up. Clin Cardiol 24:151-158

9. Gulliford M, Latinovic R (2004) Mortality in type 2 diabetic subjects prescribed metformin and sulphonylurea drugs in combination: cohort study. Diabetes Metab Res Rev 20:239245

10. Evans JMM, McDevitt DG, MacDonald TM (1995) The Tayside Medicines Monitoring Unit (MEMO): a record-linkage system for pharmacovigilance. Pharm Med 9:177-184

11. Morris AD, Boyle DIR, MacAlpine R et al, for the DARTS/ MEMO Collaboration (1997) The diabetes audit and research in Tayside Scotland (DARTS) study: electronic record-linkage to create a diabetes register. Br Med J 315:524-528

12. World Health Organization (1978) Manual of the international statistical classification of diseases, injuries and causes of death. Ninth revision. World Health Organization, Geneva

13. World Health Organization (1994) International statistical classification of diseases and related health problems. Tenth revision. World Health Organization, Geneva

14. Carstairs V (1990) Deprivation and health in Scotland. Health Bull (Edinb) 48:162-175

15. Sasali A, Leahy JL (2003) Is metformin cardioprotective? Diabetes Care 26:243-244

16. Haffner SM, Lehto S, Ronnemaa T, Pyorala K, Laakso M (1998) Mortality from coronary heart disease in subjects with type 2 diabetes and in nondiabetic subjects with and without myocardial infarction. New Engl J Med 339:229-234 